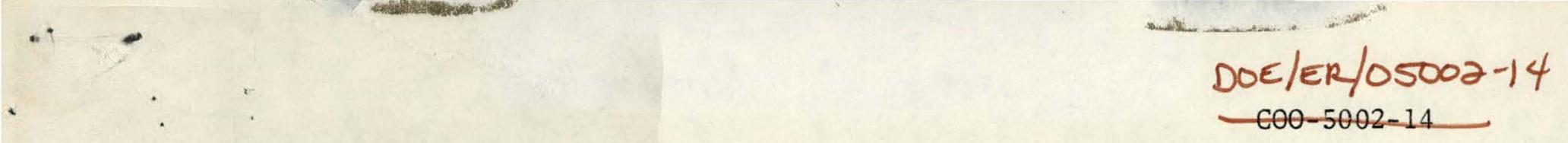

GROUP REPRESENTATION OF BICRYSTAL INVARIANT TRANSLATIONS

WITH AN APPLICATION TO THE TOPOLOGY

OF SECONDARY GRAIN BOUNDARY DISLOCATIONS

\author{
A. Brokman* \\ Department of Materials Science and Engineering \\ Massachusetts Institute of Technology \\ Cambridge, Massachusetts 02139
}

\begin{abstract}
September 1980
Massachusetts Institute of Technology

Cambridge, Massachusetts 02139

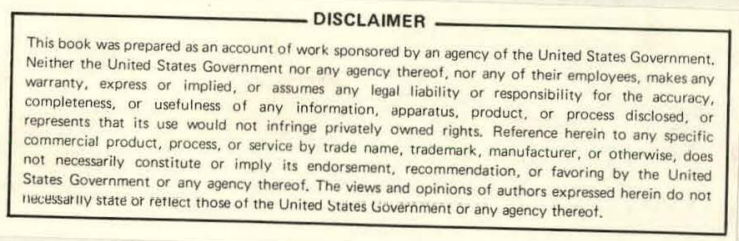

Prepared for

U. S. Department of Energy

under Contract DE-AS02-78ER05002.A001
\end{abstract}

\begin{abstract}
DISTRIBUTION OF THIS DOCUMENT IS UHLIMITER
This report was prepared as an account of work sponsored by the United States Government. Neither the United States nor the United States Department of Energy, nor any of their employees, nor any of their contractors, subcontractors, or their employees, makes any warranty, express or implied, or assumes any legal liability or responsibility for the accuracy, completeness, or usefulness of any information, apparatus, product or process disclosed or represents that its use would not infringe privately owned rights.
\end{abstract}

* Also at Bergman School for Applied Science, Hebrew University of Jerusalen, Israel. 


\section{DISCLAIMER}

This report was prepared as an account of work sponsored by an agency of the United States Government. Neither the United States Government nor any agency Thereof, nor any of their employees, makes any warranty, express or implied, or assumes any legal liability or responsibility for the accuracy, completeness, or usefulness of any information, apparatus, product, or process disclosed, or represents that its use would not infringe privately owned rights. Reference herein to any specific commercial product, process, or service by trade name, trademark, manufacturer, or otherwise does not necessarily constitute or imply its endorsement, recommendation, or favoring by the United States Government or any agency thereof. The views and opinions of authors expressed herein do not necessarily state or reflect those of the United States Government or any agency thereof. 


\section{DISCLAIMER}

Portions of this document may be illegible in electronic image products. Images are produced from the best available original document. 


\title{
GROUP REPRESENTATION OF BICRYSTAL INVARIANT TRANSLATIONS \\ WITH AN APPLICATION TO THE TOPOLOGY \\ OF SECONDARY GRAIN BOUNDARY DISLOCATIONS
}

\author{
A. Brokman
}

Department of Materials Science and Engineering

Massachusetts Institute of Technology

Cambridge, Massachusetts 02139

\begin{abstract}
A11 DSC-Latice translations of one lattice with respect to the second lattice of a bicrystal are described as a group. It is shown that the matrix representation of this group can be used to solve topological problems connected with secondary grain boundary dislocations (SGBDs) such as finding the step in the boundary associated with the SGBD. We have formulated this problem by establishing the "step vector" $\vec{S}$ associated with the Burgers vector $\vec{b}$ of the SGBD in a cubic bicrysta1. The problem is then solved in $2 \mathrm{D}$, and the way to generalize to $3 \mathrm{D}$ is indicated.
\end{abstract}




\section{INTRODUCTION}

It is now well established that secondary grain boundary dislocations (SGBDs) can exist in many high angle grain boundaries. There is considerable evidence that such SGBDs are of importance in the understanding of intrinsic grain boundary structure and energy (Balluffi, 1979) and that they also play essential roles in a number of grain boundary kinetic phenomena. For example, regular arrays of intrinsic SGBDs generally form in boundaries possessing misorientations which differ from special misorientations corresponding to a relatively high degree of coincidence between the two crystals adjoining the boundary. The role of the SGBDs in such cases is to produce a "fit-misfit" type of grain boundary structure consisting of the SGBDs embedded in the low energy interface corresponding to the nearby high coincidence misorientation. In the kinetic phenomena SGBDs have been used as the basis for the action of grain boundaries as sources/sinks for point defects (Balluffi, 1980) and as the basis for grain boundary migration and sliding (Crussard and Tamhankar, 1958; Smith and Rae, 1979). Also, a model of "diffusion induced grain boundary migration" has been proposed by Balluffi and Cahn (1980) which is based on the climb of SGBDs.

In view of this, a strong interest (King and Smith, 1980) has developed with respect to the topology of SGBDs. It is well recognized that steps in the boundary are generally associated with SGBDs, and since all of the above kinetic models are involved with these steps there is special interest in their topology. A basic question related to this is the possible step heights, which are associated with a given SGBD possessing a definite Burgers vector. The analytical solution of this problem has not yet been obtained although it has been discussed in the literature (King and Smith, 1980; Pond, 1977). In the present paper we analyze the atomic structure of a 
bicrystal and obtain a solution of this problem using a convenient algebraic group based on DSC-Lattice translations.

2. THE DSC-LATTICE FRAME AND THE CONSTRUCTION OF THE GRAIN BOUNDARY STEP Using the definition of the DSC-Lattice* it has been shown that a perfect SGBD possesses a Burgers vector which is a DSC-Lattice vector. Therefore we perform our analysis in the DSC-Lattice framework. Methematically, this framework, as will be shown, is convenient because lattice points of Crystals 1 and 2 (of the bicrystal ${ }^{* *}$ ) can be represented as the congruent class modulo $\Sigma$. Conventionally, $\Sigma$ is the volume of the repeating unit in atomic volume units (i.e., the volume of the Coincidence Site Lattice (CSL) ce11). Following Balluffi (1980), Fig. I shows how to form a SGBD and its associated step in a simple $\sum=5\left(36.9^{\circ}\right)$ symmetric tilt boundary. We start with a perfect bicrystal (Fig. 2a), make a cut along the boundary (Fig. 2b), and then translate one crystal with respect to the other by a DSC-Lattice vector (Fig. 2c). forming a SGBD. In Fig. 1c both the SGBD with its specified DSC-Lattice Burgers vector and its accompanying step can be readily identified in the DSC-Lattice frame. One way to characterize the step is to define the smallest vector $\vec{s}$ which goes from a Lattice 1 point on the right side of the step to the nearest Lattice 1 point with the same environment on the left side of the step (see Fig. 2c). The corresponding step in Lattice 2 can be obtained by adding the Burgers vector to $\vec{S}$. The

* All displacements of Lattice 2 (of the bicrystal).with respect to Lattice 1 which cause a pattern shift which is complete.

** The term "lattice of Crystal 1 (2)" refers to the mathematically infinite set of points which describes one of the two lattices of the bicrystal. "Bilattice" means the union set of the lattice of Crystal 1 set and the lattice of Crystal 2 set. 
projection of this vector on a normal to the boundary plane $(\vec{n}$ in Fig. 1c) is defined to be the step height. We can now represent $\vec{S}$ in a standard Reference Lattice which we take to be a cell of the CLS and draw $\vec{S}$ from a CSL point to the appropriate DSC-Lattice point within the cell as in Fig. 1d. This procedure has been used by other authors (King and Smith, 1980). The question now is what value of $\vec{S}$ is associated with a DSC translation $\vec{b}$ ?

Al1 of the above considerations are purely geometrical, assuming two rigid lattices (no relaxation) and based on the set of all translations (Burgers vectors) of one crystal with respect to the other under which the boundary structure remains unchanged. We shall now show that this set of "invariant translations" forms a group which can be represented in a matrix form.

\section{THE BICRYSTAL AND TRANSLATIONS GROUP REPRESENTATION}

\subsection{Representation of the Lattice Points in the DSC-Lattice Frame in} Two Dimensions

To begin, we consider a $2 D$ single CSL cell, divided into $\Sigma$ oqually spaced rows and columns parallel to the cell edges to form the DSC-Lattice, and take the coincidence site to be the origin. In each row/column there is exactly one lattice site of each crystal of the two crystals forming the bicrystal. Therefore, it is possible to define the lattice point positions of one of the two crystals, say Lattice 1, within the CSL cell as a set $\alpha$ of $\Sigma$ elements ("Lattice 1 set"):

$$
\alpha^{1}=\left\{\alpha_{m}^{1}\right\}_{m=0}^{\sum-1}
$$

where $\alpha_{m}^{1}$ is the number of the column (counting from the origin) that the 
atom occupies in the $\mathrm{m}$-th row. The Lattice $\mathrm{I}$ set of the $\Sigma=13$ boundary which runs along the $(3,2)$ direction (internal Lattice 1 coordinates) in this form is

$$
(0,8,3,11,6,1,9,4,12,7,2,10,5)
$$

(see contents of square CSL cell in lower left of Fig..2).

The set $\alpha^{1}$ can be constructed in the manner illustrated graphically in Fig. 2. Instead of taking the conventional square CSL cell we construct a linear coincidence cell which consists of $\sum$ lattice points distributed along a line between two conicidence sites.

If $(r, s)$ are the integers which generate the CSL so that

$$
\Sigma=\mathrm{r}^{2}+\mathrm{s}^{2} \quad\left(\mathrm{r}^{2}+\mathrm{s}^{2}=\text { odd }\right)
$$

(Bollmann, 1970), the linear CSL chain runs along the $(r, s)$ direction in the DSC-Lattice internal coordinate system as seen in Fig. 2 . On this linear chain a Lattice 1 point is found every $r$ columns or $s$ rows of the DSC-Lattice frame. This chain can be" "roduced" into the conventional square CSL cell by the graphical procedure illustrated in Fig. 2. It may be seen that the translation of each Lattice 1 point consists of an integral number of $\Sigma$ DSC-Lattice rows and columns. Mathematically, this operation can be presented by the congruence modulo $\Sigma$ class: Let $\mathrm{m}$ be the row number (counting from the origin) and $\mathrm{n}$ an integer less than $\Sigma$. If:

$$
\mathrm{m} \equiv \operatorname{ns}(\bmod \Sigma),
$$

then the Lattice 1 set $\mathrm{m}$-th element is:

$$
\alpha_{\mathrm{m}}^{1} \equiv \operatorname{nr}(\bmod \Sigma)
$$

while the Lattice 2 set (we imagine two penetrating lattices) is: 


$$
\alpha_{\mathrm{m}}^{2} \equiv-\operatorname{nr}(\bmod \Sigma)
$$

Having obtained the frame with the two lattice sets, SGBDs with DSC-Lattice Burgers vector $\vec{b}=(p, q)(q, p$ integers in units of the DSC-Lattice frame) are introduced by adding $p$ to each member of the set and cyclically shifting each element by $q$ places to the right in the set. The new set, after these operations, will be defined for

$$
m=(n+q)(\bmod \Sigma)
$$

as

$$
\alpha_{m}=\left(\alpha_{n}+p\right)(\bmod \Sigma)
$$

\subsection{Rotation and Translation in the DSC-Lattice Frame}

So far, we have described the geometry of the two lattices in the DSC-Lattice frame, and have introduced the translation in this frame. Because both rotation and translation are represented here in terms. of a permutation of the $(0,1 \ldots \Sigma-1)$, two sets of operators above the $\Sigma$ ! order permutationgroup are of interest:

(a) The set of permutation operators which is associated with all allowed $(r, s)$ rotations, namely, $x_{r, s^{*}}^{\Sigma}$. These operators act on the $(0,1,2 \ldots \Sigma-1)$ element of the group (the identity element) according to Eqs. (1a) and (1b), thus giving the permutation of the Lattice 1 set. We designate as $\left(x_{r, s}^{\Sigma}\right)^{t}$ the operation that gives the second lattice set [Eq. (1c)].

(b) The set of permutation operators which is associated with all translations $(p, q)$, i.e., Burgers vectors $)$, is designated $s_{p, q}^{\Sigma}$ and acts according to Eq. (2). 
In this manner the group of operators $\{S x\}$, for all possible $(r, s)$ and $(p, q)$, forms an operator space above the $\Sigma$ ! order permutation group. Working in this space rather than in the regular geometrical rotation/translation space has the advantage that the operators contain implicitly the information about the location of lattice points.

\subsection{The Rotation Representation}

One convenient way to handle the two sets is to look at the matrix representation of them; we construct the $\sum \times \Sigma$ matrix of $\chi_{r, s}^{\Sigma}$ from Eq. (1a):

$$
\left(\chi_{r, s}^{\Sigma}\right)_{i, j}=\delta_{i-1, n r(\bmod \Sigma)} \delta_{j-1, n s(\bmod \Sigma)} \cdot(\forall n)
$$

This way the matrix element $(i, j)$ is equal to unity if the associated (column, row) of the DSC-Lattice frame is occupied by a Lattice 1 point, and zero otherwise (see footnote in Section 3.4). In the same manner, the transposed matrix $\left(\chi_{r, s}^{\Sigma}\right)^{t}$ spans the second lattice. For example, we take the $\Sigma=13$ case where $r=3, s=2$ (our examples are for $\Sigma=13$ in order not to he restricted to the case $s=1$. which might be misleading because then $\mathrm{m}=\mathrm{n}$ ). Then,

$$
\times_{3,2}^{1.3}=\left[\begin{array}{lllllllllllll}
1 & 0 & 0 & 0 & 0 & 0 & 0 & 0 & 0 & 0 & 0 & 0 & 0 \\
0 & 0 & 0 & 0 & 0 & 0 & 0 & 0 & 1 & 0 & 0 & 0 & 0 \\
0 & 0 & 0 & 1 & 0 & 0 & 0 & 0 & 0 & 0 & 0 & 0 & 0 \\
0 & 0 & 0 & 0 & 0 & 0 & 0 & 0 & 0 & 0 & 0 & 1 & 0 \\
0 & 0 & 0 & 0 & 0 & 0 & 1 & 0 & 0 & 0 & 0 & 0 & 0 \\
0 & 1 & 0 & 0 & 0 & 0 & 0 & 0 & 0 & 0 & 0 & 0 & 0 \\
0 & 0 & 0 & 0 & 0 & 0 & 0 & 0 & 0 & 1 & 0 & 0 & 0 \\
0 & 0 & 0 & 0 & 1 & 0 & 0 & 0 & 0 & 0 & 0 & 0 & 0 \\
0 & 0 & 0 & 0 & 0 & 0 & 0 & 0 & 0 & 0 & 0 & 0 & 1 \\
0 & 0 & 0 & 0 & 0 & 0 & 0 & 1 & 0 & 0 & 0 & 0 & 0 \\
0 & 0 & 1 & 0 & 0 & 0 & 0 & 0 & 0 & 0 & 0 & 0 & 0 \\
0 & 0 & 0 & 0 & 0 & 0 & 0 & 0 & 0 & 0 & 1 & 0 & 0 \\
0 & 0 & 0 & 0 & 0 & 1 & 0 & 0 & 0 & 0 & 0 & 0 & 0 \\
& & & & & & & & & & & &
\end{array}\right]
$$


The lattice sets can be obtained by simple matrix operations:

$$
\begin{aligned}
& \alpha^{1}=x_{2,1}^{13} \alpha_{I}=(0,8,3,11,6,1,9,4,12,7,2,10,5), \\
& \alpha^{2}=\left(x_{2,1}^{13}\right)^{t} \cdot \alpha_{I}=(0,5,10,2,7,12,4,9,1,6,11,3,8) .
\end{aligned}
$$

Here, $\alpha_{I}$ is the column of the "identity" element of the permutation group, $(0,1,2,3 \ldots \ldots 12)$.

\subsection{The Translation Group Representation}

We now construct the matrix $S_{p, q}$ which is associated with the translation $(p, q)$ by recognizing the way in which it shifts the $x_{r, s}$ matrix element:

$$
\begin{aligned}
\left(\mathrm{T}_{\mathrm{p}, q, r, s}^{\Sigma}\right)_{i, k}= & \sum_{j=1}^{\sum}\left(s_{p, q}^{\Sigma}\right)_{i, j}\left(\chi_{r, s}^{\Sigma}\right)_{j, k}= \\
& \left(\chi_{r, s}^{\Sigma}\right)_{(i+p)(\bmod \Sigma),(j+q)(\bmod \Sigma)}
\end{aligned}
$$

All of the right hand cyclic permutations $T_{p, q, r, s}^{\Sigma} \alpha_{I}$ of a given $(r, s)$ form a subgroup of the permutation group, which might be called "the $(r, s)$ lattice translation group" because it represents all possible translations of Lattice 1 with respect to Lattice 2 under which the bi-lattice structure is invariant. We are now interested in the matrix representation of this group.

Naturally, we take the non-translated $\mathrm{T}_{0,0, \mathrm{r}, \mathrm{s}}^{\Sigma}$ to be $\chi_{\mathrm{r}, \mathrm{s}}^{\Sigma}$, i.e., $S_{n, n}=I$, where $I$ is the identity matrix. According to Eq. (1), the $S_{p, q}$ matrix representation can be constructed by the $\left(p+q \alpha_{\Sigma-1}\right) \cdot(\bmod \Sigma)$ units to the right. The assumption here is that a unit column translation induces $\alpha_{\Sigma-1}$ unit row translations as shown in Fig. 3. This conclusion is derived from the following argument: 
We look at the "Lattice 1 set" $\alpha^{1}$, and search for the number that we have to add to each element of the set in order to obtain the same group element as if we had cyclically shifted the whole set by one unit to the right. Because $\alpha_{1}=0$, and after the shifting $\alpha_{1}^{\prime}=\alpha_{\Sigma-1}\left(\alpha_{1}^{\prime}\right.$ is the value of $\alpha_{1}$ after the shift), the additive number that we are seeking is $\alpha_{\Sigma-1}$. We discuss in the Appendix the behavior of $\alpha_{\Sigma-1}$ and show that for small $(r, s)[r<9, s<4$ except for the couple $(4,3)]$,

$$
\alpha_{\Sigma-1} \equiv \Sigma-\left(\frac{r+1}{s} r+s\right)(\bmod \Sigma) .
$$

Other cases of larger $(r, s)$ (which are probably irrelevant because of the relatively low degree of coincidence) can be solved by Number Theory methods if necessary.

Having $\alpha_{\Sigma-1}$, the matrix representation for the Lattice I translations group element $S_{p, q}$ is given by the relation*

$$
\left.\left(s_{p, q}\right)_{i, j}=\delta_{i,(j+p+q} \alpha_{\Sigma-1}\right)(\bmod \Sigma)
$$

For example, the matrix representation of $(p, q)=(1,1)$ for $\Sigma=13$ is:

* Also, one can similarly represent $\chi$ of Eq. (3) by

$$
\left(x_{r, s}\right)_{i, j}=\delta_{i-1,-(j+1) \alpha_{\Sigma-1}(\bmod \cdot \Sigma)}
$$




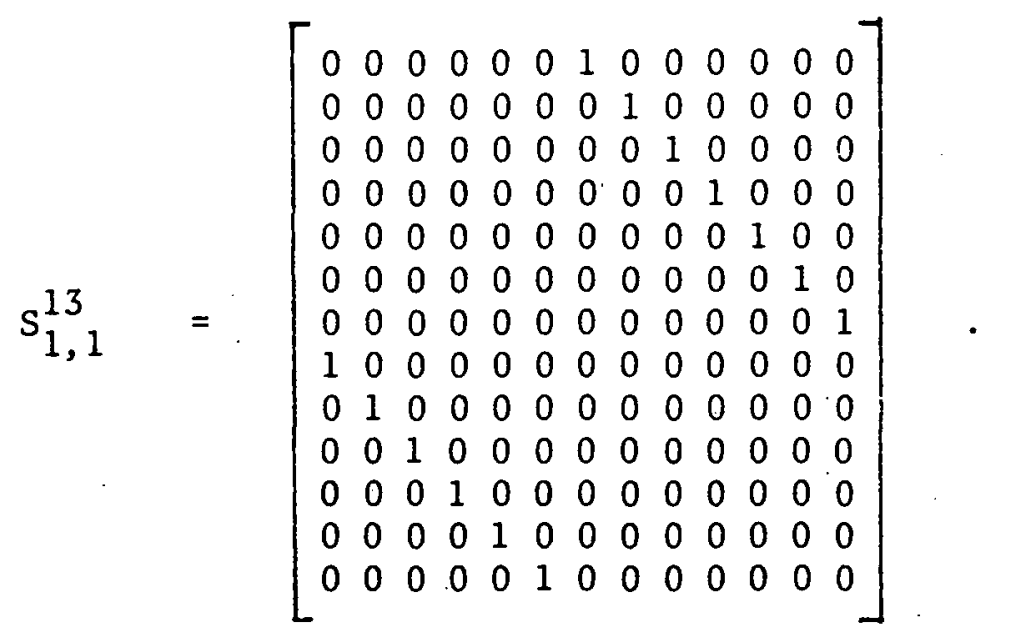

Then, by Eq. 4:

$$
\mathrm{S}_{1,1}^{13} \times_{3,2}^{13}=\left[\begin{array}{lllllllllllll}
0 & 0 & 0 & 0 & 0 & 0 & 0 & 0 & 0 & 1 & 0 & 0 & 0 \\
0 & 0 & 0 & 0 & 1 & 0 & 0 & 0 & 0 & 0 & 0 & 0 & 0 \\
0 & 0 & 0 & 0 & 0 & 0 & 0 & 0 & 0 & 0 & 0 & 0 & 1 \\
0 & 0 & 0 & 0 & 0 & 0 & 0 & 1 & 0 & 0 & 0 & 0 & 0 \\
0 & 0 & 1 & 0 & 0 & 0 & 0 & 0 & 0 & 0 & 0 & 0 & 0 \\
0 & 0 & 0 & 0 & 0 & 0 & 0 & 0 & 0 & 0 & 1 & 0 & 0 \\
0 & 0 & 0 & 0 & 0 & 1 & 0 & 0 & 0 & 0 & 0 & 0 & 0 \\
1 & 0 & 0 & 0 & 0 & 0 & 0 & 0 & 0 & 0 & 0 & 0 & 0 \\
0 & 0 & 0 & 0 & 0 & 0 & 0 & 0 & 1 & 0 & 0 & 0 & 0 \\
0 & 0 & 0 & 1 & 0 & 0 & 0 & 0 & 0 & 0 & 0 & 0 & 0 \\
0 & 0 & 0 & 0 & 0 & 0 & 0 & 0 & 0 & 0 & 0 & 1 & 0 \\
0 & 0 & 0 & 0 & 0 & 0 & 1 & 0 & 0 & 0 & 0 & 0 & 0 \\
0 & 1 & 0 & 0 & 0 & 0 & 0 & 0 & 0 & 0 & 0 & 0 & 0 \\
& & & & & & & & & & & &
\end{array}\right],
$$

which gives unity. in any row/column whenever a translated Lattice 1 point occupies a position in the DSC-Lattice frame according to the previous convention.

An analogous subgroup is the one of all left-hand cyclic permutations of $\left(\chi_{r, s}^{\sum^{*}}\right)^{t}$. Clearly, this subgroup represents all invariant translations of Lattice 2 with respect to Lattice 1 . This subgroup might be called the " $(r, s)$ Lattice 2 translations group". It is possible to represent 
this group above $\chi_{r, \mathrm{~s}}^{\Sigma}$ by a simple relation to the $s_{p, q}^{\Sigma}$ group:

$$
\left(S_{p, q}^{\Sigma}\right)_{i, j}^{t}=\left(S_{p, q}^{\Sigma}\right)(\Sigma-i+2)(\bmod \Sigma), j
$$

We define the row elements to be $(\Sigma-i+2)(\bmod \Sigma)$ so that the identity element of the first group will be transferred to the element of the second group which represents the non-translated group element. For example, $\left(x_{3,2}^{13}\right)^{t}$ in this representation will be

$$
\left(\mathrm{S}_{0,0}^{13}\right)^{\mathrm{t}}=\left[\begin{array}{lllllllllllll}
1 & 0 & 0 & 0 & 0 & 0 & 0 & 0 & 0 & 0 & 0 & 0 & 0 \\
0 & 0 & 0 & 0 & 0 & 0 & 0 & 0 & 0 & 0 & 0 & 0 & 1 \\
0 & 0 & 0 & 0 & 0 & 0 & 0 & 0 & 0 & 0 & 0 & 1 & 0 \\
0 & 0 & 0 & 0 & 0 & 0 & 0 & 0 & 0 & 0 & 1 & 0 & 0 \\
0 & 0 & 0 & 0 & 0 & 0 & 0 & 0 & 0 & 1 & 0 & 0 & 0 \\
0 & 0 & 0 & 0 & 0 & 0 & 0 & 0 & 1 & 0 & 0 & 0 & 0 \\
0 & 0 & 0 & 0 & 0 & 0 & 0 & 1 & 0 & 0 & 0 & 0 & 0 \\
0 & 0 & 0 & 0 & 0 & 0 & 1 & 0 & 0 & 0 & 0 & 0 & 0 \\
0 & 0 & 0 & 0 & 0 & 1 & 0 & 0 & 0 & 0 & 0 & 0 & 0 \\
0 & 0 & 0 & 0 & 1 & 0 & 0 & 0 & 0 & 0 & 0 & 0 & 0 \\
0 & 0 & 0 & 1 & 0 & 0 & 0 & 0 & 0 & 0 & 0 & 0 & 0 \\
0 & 0 & 1 & 0 & 0 & 0 & 0 & 0 & 0 & 0 & 0 & 0 & 0 \\
0 & 1 & 0 & 0 & 0 & 0 & 0 & 0 & 0 & 0 & 0 & 0 & 0
\end{array}\right]
$$

Now, the translations group has a convenient representation, and we will demonstrate this advantage by an application to the problem of the step vector.

\subsection{Application to the Step Topology of the SGBD}

In view of Section 1.1 , the vector $\vec{S}$ is a DSC-Lattice vector, say, $(m, n)$ where $m(n)$ is the DSC-Lattice column (row) of the coincidence point 
after the translation. The quantity $(m, n)$ can be found by identifying the element $(m, n)$ of the two matrixes $S x$ and $x^{t}$ which is equal to unity:

$$
\left(s_{p, q}^{\Sigma} x_{r, s}^{\Sigma}\right)_{m, n}=\left(x_{r, s}^{\Sigma}\right)_{m, n}^{t}=1,
$$

or,

$$
\left(s_{p, q}^{\Sigma} x_{r, s}^{\Sigma}\right)_{m, n}=\left[\left(s_{0,0}^{\Sigma}\right)^{t}\left(x_{r, s}^{\Sigma}\right)\right]_{m, n}=1 .
$$

In other words, we need the $m$ for which the non-zero element $(m, j)$ of the $(p, q)$ right hand matrix is equal to the $(m, j)$ left hand (00) matrix element. The column can be obtained, following the definition of the two matrixes [Eqs. (11) and (14)], by the equation

$$
\left(m+p+q \alpha_{\Sigma-1}\right)(\bmod \Sigma)=\left[\Sigma-\left(m+p+q \alpha_{\Sigma-1}\right)+2\right](\bmod \Sigma)
$$

Because there is only one non-zero term in each column/row of both $S$ and $\chi,(m, n)$ is defined for each $m$ by way of Eq. (3). We summarize by writing the vector $\vec{S}$ (in DSC-Lattice units) as:

$$
\vec{s}_{p, q}=\left(\begin{array}{c}
m^{\prime} \\
m^{\prime}\left(\Sigma-\alpha_{\Sigma-1}\right)(\bmod \Sigma)
\end{array}\right)
$$

where the two components of the vector are along the CSL cell edges, and $m^{\prime}=m_{-2}$ is derịved from Eq. (17) as

$$
m^{\prime}=\frac{1}{2}\left\{\left(p+q \alpha_{\Sigma-1}\right)(\bmod \Sigma)+\frac{1}{2}\left[1-(-1)\left(p+q \alpha_{\Sigma-1}\right)(\bmod \Sigma) j \Sigma\right\}\right.
$$

The quantity $\mathrm{m}^{\prime}\left(\Sigma-\alpha_{\Sigma-1}\right)(\bmod \Sigma)$ is the step height of a boundary which runs parallel to a DSC-Lattice row in Fig. Id.

In the case of Fig. 1, for example $\left(p=0, q=1, \alpha_{\Sigma-1}=3\right)$ we have from. 
Eq. (19) $\mathrm{m}^{\prime}=4$, and, therefore, from Eq. (18),

$$
\vec{S}=\left(\begin{array}{l}
4 \\
3
\end{array}\right)
$$

which is the vector that is drawn in Fig. 1d.

\section{DISCUSSION}

When a SGBD is introduced in two dimensions, a singular point at the boundary is produced between two perfect structures. This singularity is associated with the dislocation core and the accompanying step. The topological properties of these singular structures provide a basis for physical properties of the bicrystal which cannot be explained by means of regular boundary structures, for instance, the kinetic properties that were mentioned in the Introduction. However, because of their singular character, there are some problems in specifying their topology. Mathematically, in Section 3 we presented the algebraic group which might be applied to the description of the bicrystal topology: The singularity treatment required a mathematical "manipulation" by which we compared the elements of two groups: one representing translations of one crystal with respect to the second, and the other representing translations of the second crystal with respect to the first. It should be noted that this operation (of comparing elements) is not of any physical significance, and is purely for mathematical convenience. This point is emphasized here in order to avoid any misunderstandings about the physical significance of the present step treatment.

Finally, we generalize the validity of our solution for the topology of SGBDs to three dimensions. It is clear that the model for two dimensions still holds for the [100] tilt boundary in simple cubic materials. The 
generalization to [100] tilt boundaries in FCC materials. (the BCC results can be derived in the same way) can be done as follows:

For FCC materials the CSL is BCT where the base of the primitive cell is the $2 D \mathrm{CSL}$ structure that has already been discussed. Half a lattice parameter (FCC lattice coordinates) above the base the same structure appears again but translated by $(1 / 2,1 / 2) 2 \mathrm{D}-\mathrm{CS} \mathrm{L}$ units. The projection of the entire structure on the base plane, therefore, produces a framework which is four times as dense as the 2D DSC-Lattice as seen in Fig. 4. For analyzing the [100] tilt boundary step it is enough to know the height of the step in this frame. For this reason the discussion of Section 3 can be applied, but, instead of describing the lattice set as $\left\{-n \alpha_{\Sigma-1}(\bmod \Sigma)\right\}_{n=0}^{\Sigma-1}$; we take it to be $\left[-n \alpha_{\Sigma-1}(\bmod 2 \Sigma)\right\}_{n=0}^{2 \Sigma-1}$. (We have here used $\alpha_{\Sigma-1}$ for abbreviation; it corresponds to the $\alpha_{\Sigma-1}$ element of the 2D set.) From here on we can follow the arguments of Section 3 and obtain the projection of the step vector on the CSL base plane. The third component of the step is. just the third component of the Burgers vector $(\bmod 2)$.

The same arguments of Section 3 hold for any other [hkl] rotation axis used to produce the CSL while using the expression $\Sigma=r^{2}+\left(h^{2}+k^{2}+l^{2}\right) s^{2}$ (Ranganathan, 1966).

An algebra of the translation group can be constructed by the collection of all the operators:

$$
A=\Sigma a_{p, q} \hat{S}_{p ; q},
$$

where $a_{p, q}$ is a scalar and $\hat{S}_{p, q}$ is defined by

$$
\hat{S}_{p, q} \phi(\vec{r})=\phi(\vec{r}+\vec{b})
$$


for any physical property $\phi$ which is dependent on the spatial group of $\vec{r}$. We have restricted ourselves to the topology of bicrystals in the present paper; therefore, we used $\phi(\vec{r})=\vec{r}$, but the discussion can be generalized. In Section. 3.4 it was shown that there are only $\Sigma$ operators above the translations group. This may be surprising because the DSC-Lattice frame contains $\Sigma \times \Sigma$ possible translations. The physical reason for this is that there are only $\Sigma$ coincidence sites which can be produced by translations of Lattice 1 with respect to Lattice 2. For this reason we should write Eq. (20) as

$$
A=\sum_{i=1}^{\sum} a_{i} \hat{s}_{i}
$$

In the previous section, $\hat{S}_{i}$ is the group of all DSC-Lattice translations in the $x$ direction in one CSL. Another convenient set, $\hat{S}_{i}$, is all the translations from the origin to the second lattice points. Because we can get any invariant pattern with this algebra, we can use the $\Sigma$ translations of the group $S_{i}$ for describing the DSC-Lattice. This might be important when dealing with 3D translations because this group has cubic symmetry. 
5. SUMMARY

The crystallography of SGBDs can be presented by a group with a simple matrix representation. This gives mathematical tools for dealing with the topology of SGBDs and predicting the step vector $\vec{S}$ which is associated with their Burgers vectors $\vec{b}$. This group can also be used for any other physical property of the translated bicrystal using the algebra defined by the equation (22).

\section{ACKNOWLEDGMENT}

Support was provided by the U.S. Department of Energy under Contract DE-AS02-78ER05002.A001. The author would like to thank Professor R. W. Balluffi and Drs. P. D. Bristowe and A. H. King for their comments. 


\section{REFERENCES}

Balluffi, R. W. (1979). In Interfacial Segregation, ed. by W. C. Johnson and J. M. Blakely, ASM, Metals Park, Ohio, p. 193.

Balluffi, R. W. (1980). Paper presented at 1979 ASM Materials Science Seminar on Grain Boundary Structure and Kinetics, 15-16 September, Milwaukee, Wisconsin (to be published by ASM, Metals Park, Ohio).

Balluffi, R. W. and Cahn, J.W. (1980). To be published.

Bollmann, w. (1970). Crystal Defects and:Crystalline Interfaces. Berlin: Springer, p. 145.

Crussard, C. and Tamhankar, R. (1958). Trans. Metall. Soc., AIME 212, 718.

King, A. H. and Smith, D. A. (1980). Acta Cryst. A36, 335.

Mathews, G. B. (1965). Theory of Numbers, 2nd ed., New York: Chelsea, p. 53.

Pond, R. C. (1977). Proc. R. Soc. London Ser. A 357, 471.

Ranganathan, S. (1966). Acta Cryst. 21, 197.

Smith, D. A. and Rae;.C. M. F. (1979). Met. Sci. J. 13, 101. 


\section{APPENDIX}

A similar problem is finding the value of $\alpha_{1}\left(\alpha_{\Sigma-1}=\sum-\alpha_{1}\right)$ :

For each lattice point on the linear chain (mr, ms) [DSC-Lattice coordinates; see the description of the linear CSL in Section 3.1], there is a lattice point at $\left(\mathrm{mr}+\ell_{1} s\right.$, ms $\left.-\ell_{1} r\right) \cdot\left(\ell_{i}\right.$ integer $)$. In our notation the column value is the $\alpha_{n}$ for row $n$; therefore, we write for $\alpha_{1}$ the two equations:

$$
\begin{aligned}
\alpha_{1} & =\mathrm{mr}+\ell_{1} s, \\
1 & =\mathrm{ms}-\ell_{2} r,
\end{aligned}
$$

or, after translating to the square CSL cell, $\left(l_{1} l_{2}=1\right)$ :

$$
\alpha_{1}=\left(\frac{r+1}{s} r+s\right)(\bmod \Sigma)
$$

The condition that we assumed here, $r(r+1)=0(\bmod \Sigma)$ is true for any $(r, s)$ which are smaller than $(9,4)$ except $(4,3)$. Another wa Another way of finding $\alpha_{\Sigma-1}$ (which can be applied to the cases of high indices and $\ell_{1}, l_{2}$ different from 1 ) is by writing the two equations for rows and columns:

(a) The differencc in rows is 1 :

$$
-1=\operatorname{ms}(\bmod \Sigma) \quad ;
$$

(b) The difference in columns is $\alpha_{\Sigma-1}$ :

$$
\alpha_{\Sigma-1}=\operatorname{mr}(\bmod \Sigma)
$$

Squaring and adding both identities (remember $r^{2}+s^{2}=\Sigma$ ) gives the diophantic equation:

$$
\alpha_{\Sigma-1}^{2}=-1(\bmod \Sigma) \text {, }
$$

which can be solved using Number Theory (Mathews, 1965): 
Fig. 1. Construction of the SGBD: starting from the perfect symmetric [310] tilt boundary (a), producing a gap $\vec{d}$ which is a lattice vector of the DSC-Lattice (b), and then closing the gap to get an edge SGBD wi.th Burgers vector $\vec{d}$ and a step vector $\vec{S}$ (c). $\vec{S}$ goes from the coinçidence site before the translation to a coincidence site after the translation (d).

Fig. 2. Construction of conventional CSL from the linear CSL for a $\Sigma=13(\mathrm{r}=3, \mathrm{~s}=2)$ boundary.

Fig. 3. Example of the relation between column and row translation in $\Sigma=13(\mathrm{r}=3, \mathrm{~s}=2)$ CSL cell. A translation by one DSC-Lattice parameter induces a translation of $\alpha_{\Sigma-1}$ DSC-Lattice parameters in the perpendicular direction.

Fig. 4. The projection of the second plane of the $3 D$ primitive $\Sigma=13$ CSL cell on the first plane for a FCC material. We can apply the Section 3 formalism to this structure by using a $2 \Sigma \times 2 \sum$ DSC-Lattice frame, because in each column/row there is only one lattice point of each crystal of the bicrystal. 


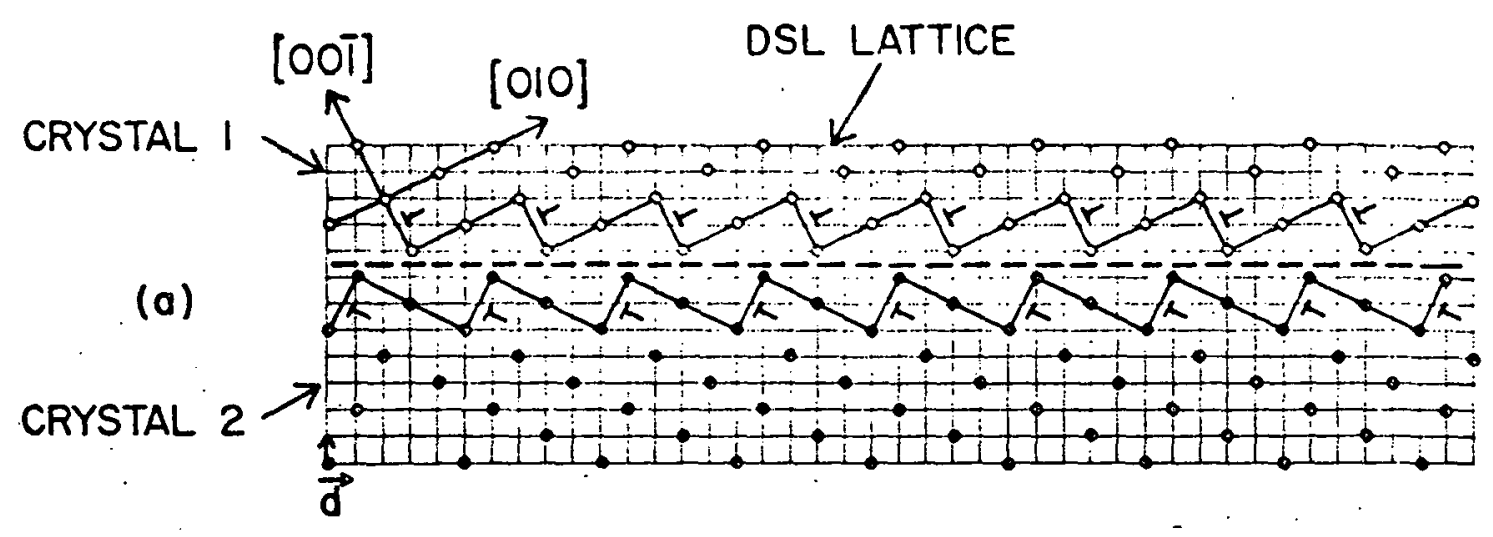

(b)

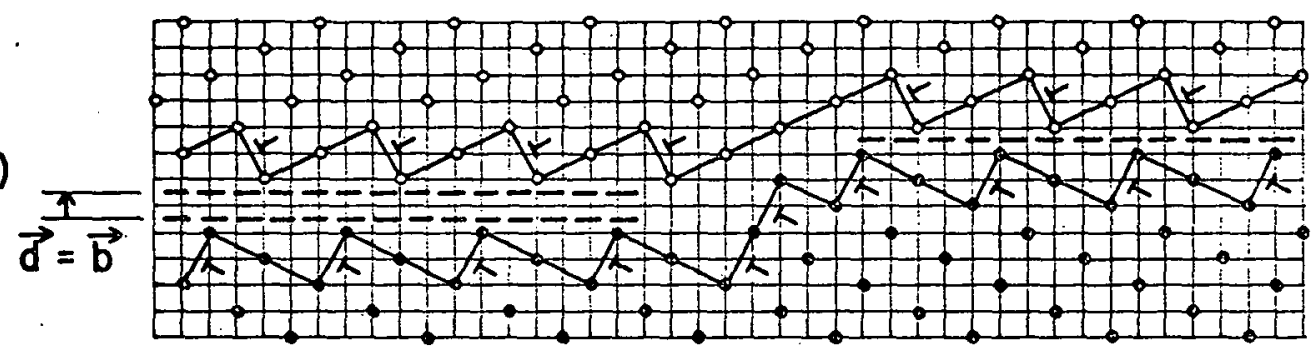

(c)

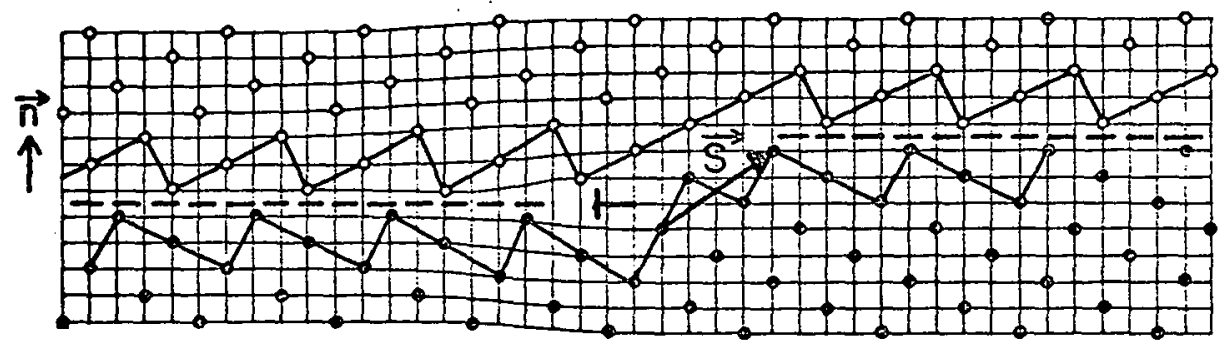

(d)

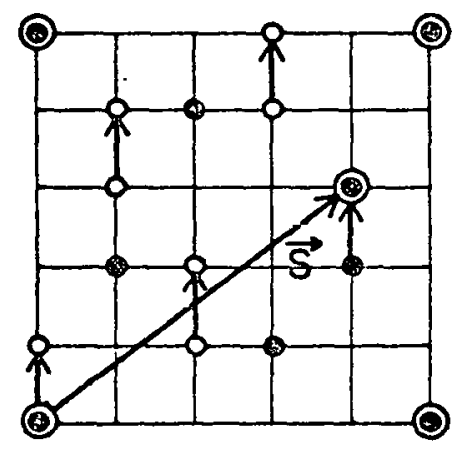

FIG. I 


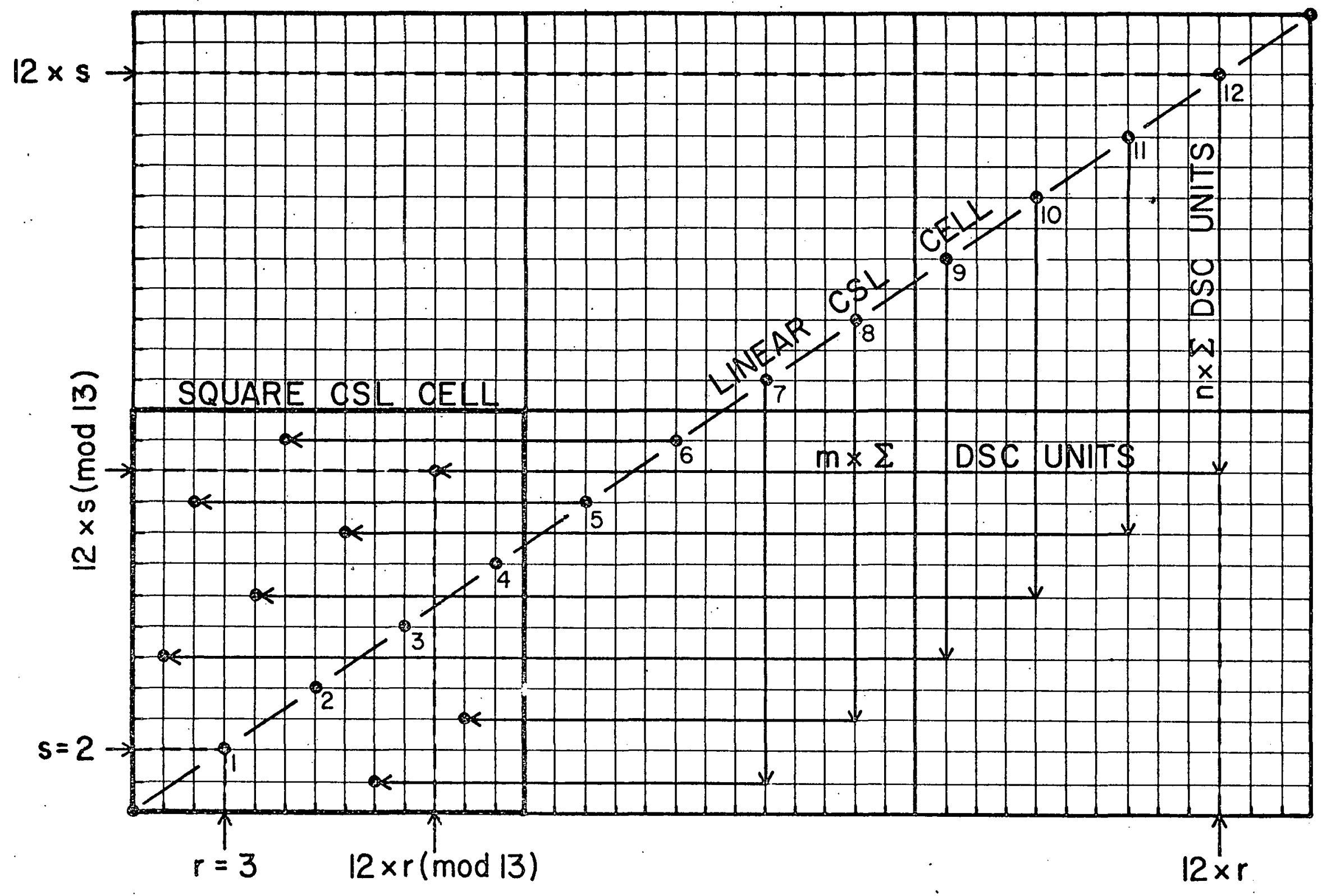

FIG2 


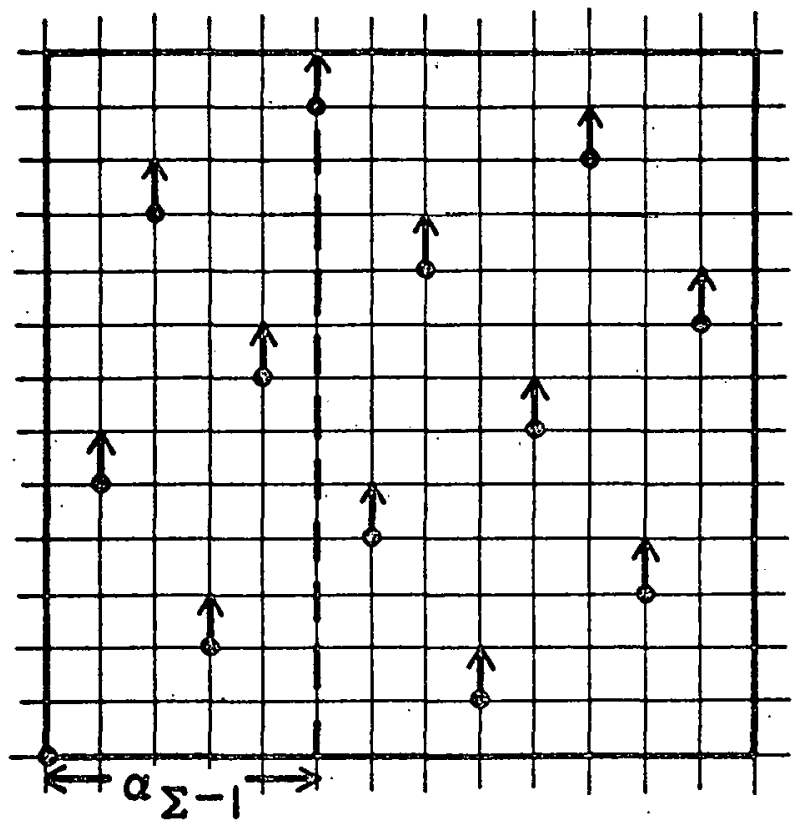

(a)

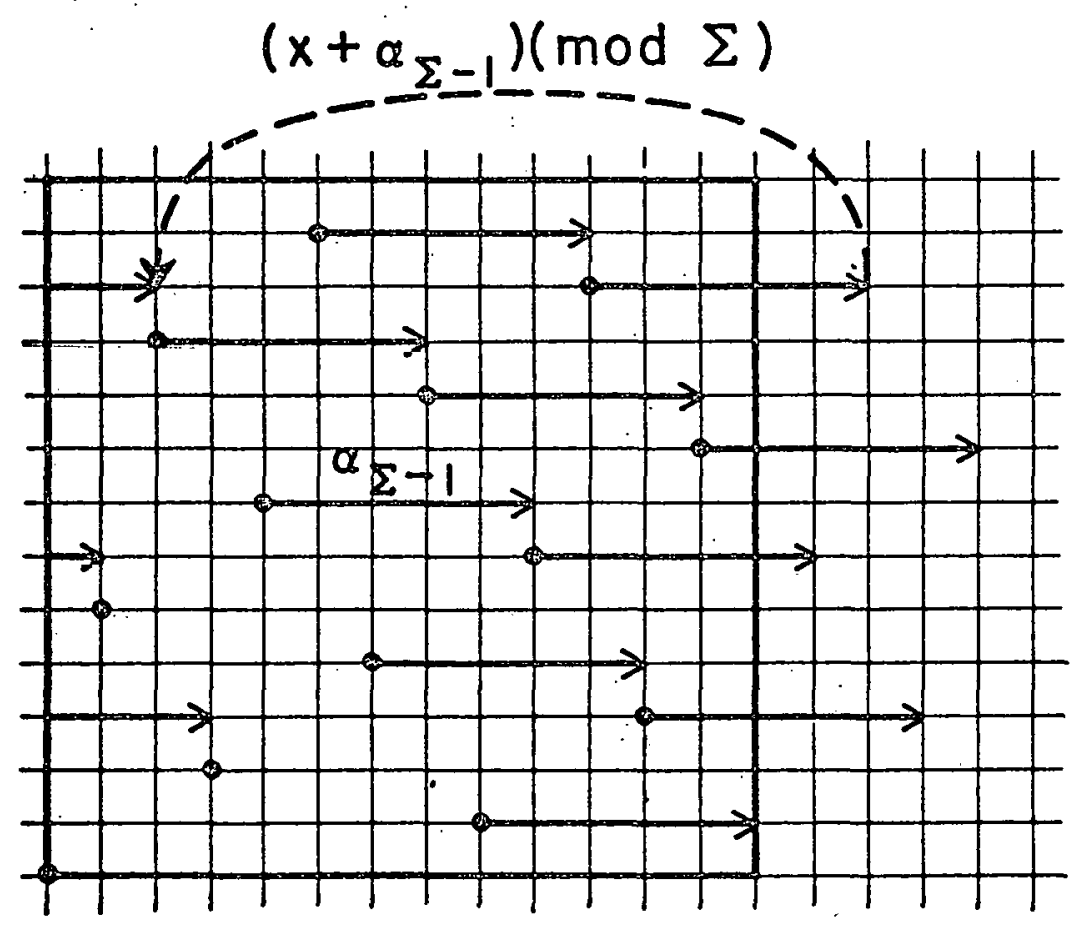

(b) 


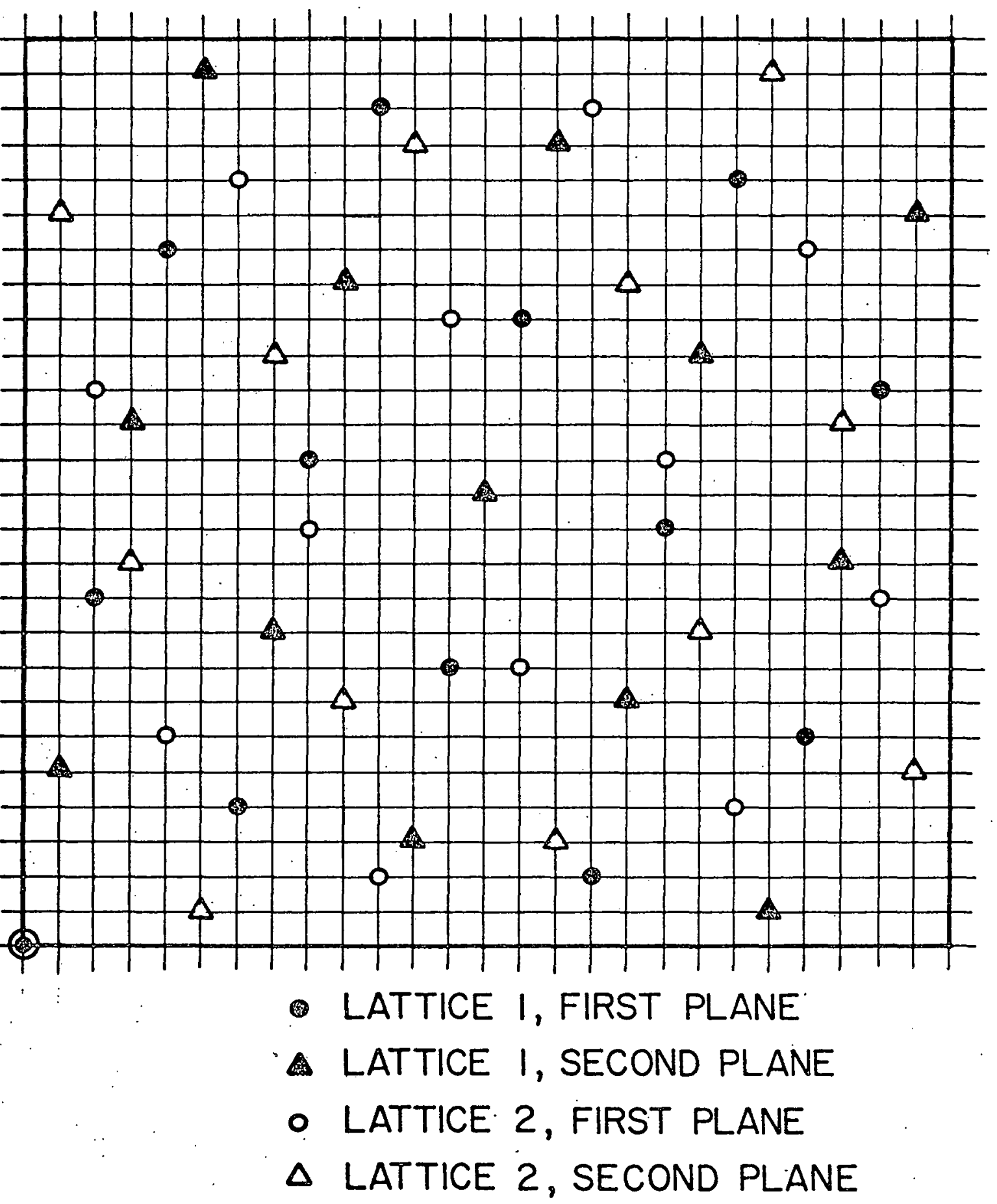

